\title{
A Novel Robot-Assisted Kinematic Measure for Children with Attention-Deficit/Hyperactivity Disorder: A Preliminary Study
}

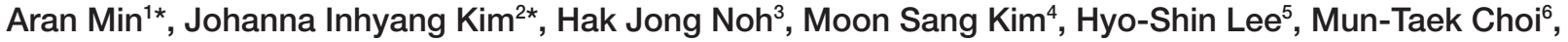 \\ Kyuha Lee ${ }^{7}$, Jun-Ho Seo ${ }^{8}$, Ga Hyun Lee ${ }^{9}$, Seong-kyu Kang ${ }^{3}$, and Dong Hyun Ahn ${ }^{2,9,10} \bowtie$ \\ ${ }^{1}$ Bodyfriend, Incorporated, Seoul, Republic of Korea \\ ${ }^{2}$ Department of Psychiatry, Hanyang University Medical Center, Seoul, Republic of Korea \\ ${ }^{3}$ Robocare, Co., Seongnam, Republic of Korea \\ ${ }^{4}$ Department of Biomedical Science and Engineering, Gwangju Institute of Science and Technology, Gwangju, Republic of Korea \\ ${ }^{5}$ Department of Early Childhood Special Education, Daegu University, Gyeongsan, Republic of Korea \\ ${ }^{6}$ School of Mechanical Engineering, Sunkyunkwan University, Suwon, Republic of Korea \\ ${ }^{7}$ Wedu Communications, Incorporated, DMC Hi-tech Industry Center, Seoul, Republic of Korea \\ ${ }^{8}$ Korean Association of Robot Industry, Seoul, Republic of Korea \\ ${ }^{9}$ Department of Child Psychotherapy, Hanyang University College of Medicine, Seoul, Republic of Korea \\ ${ }^{10}$ Department of Psychiatry and Institute of Mental Health, Hanyang University College of Medicine, Seoul, Republic of Korea
}

Objective Attention-deficit/hyperactivity disorder (ADHD) is a neurodevelopmental disorder characterized by inattention, hyperactivity, and impulsivity. In contrast to neurocognitive measurements of inattention and impulsivity, there has been limited research regarding the objective measurement of hyperactivity in youths with ADHD. The purpose of the present study was to investigate the clinical effectiveness of a newly developed Robot-assisted Kinematic Measure for ADHD (RAKMA) in children with ADHD.

Methods In total, 35 children with ADHD aged 5 to 12 years and 50 healthy controls (HCs) were recruited, and the parents completed the Child Behavior Checklist and the Korean ADHD Diagnostic Scale. RAKMA performance was represented by RAKMA stimulusresponse and hyperactivity variables. We compared the RAKMA performance of those with ADHD and with that of HCs and also investigated the correlation between the RAKMA variables and ADHD clinical scale scores.

Results Significant differences between the ADHD and HC groups were observed regarding most RAKMA variables, including correct reactions, commission errors, omission errors, reaction times, migration distance, and migration speed scores. Significant correlations were detected between various ADHD clinical scale scores and RAKMA variables.

Conclusion The RAKMA was a clinically useful tool for objectively measuring hyperactivity symptoms in children with ADHD. Further studies with larger samples are warranted.

Psychiatry Investig 2021;18(7):645-651

Key Words ADHD, Clinical utility, Hyperactivity, Kinematic analysis, Robot.

\section{INTRODUCTION}

Attention-deficit/hyperactivity disorder (ADHD) is a common neurodevelopmental disorder whose core symptoms include developmentally inappropriate levels of inattention, impul-

Received: February 4, 2021 Revised: April 15, 2021

Accepted: April 23, 2021

$\triangle$ Correspondence: Dong Hyun Ahn, MD, PhD

Department of Psychiatry and Institute of Mental Health, Hanyang University College of Medicine, 222 Wangsimni-ro, Seongdong-gu, Seoul 04763, Republic of Korea

Tel: +82-2-2292-8425, Fax: +82-2-2292-2055, E-mail: ahndh@hanyang.ac.kr

*These authors contributed equally to this work.

(a) This is an Open Access article distributed under the terms of the Creative Commons Attribution Non-Commercial License (https://creativecommons.org/licenses/by$\mathrm{nc} / 4.0$ ) which permits unrestricted non-commercial use, distribution, and reproduction in any medium, provided the original work is properly cited. sivity, and hyperactivity. ${ }^{1} \mathrm{ADHD}$ was previously called attentiondeficit disorder in the Diagnostic and Statistical Manual of Mental Disorders III (DSM-III) in 1980 but was renamed ADHD in the Diagnostic and Statistical Manual of Mental Disorders III-Revised (DSM-III-R) in 1987. ${ }^{2}$ The International Statistical Classification of Diseases and Related Health Problems 10th edition $^{3}$ uses the specific diagnostic term of hyperkinetic disorder to describe the syndrome of ADHD. ${ }^{4}$ Despite the centrality of hyperactivity in the diagnosis of ADHD, research on objective tests measuring hyperactivity has been very limited. ${ }^{5}$ The continuous performance test (CPT) has been widely used as an objective test to assess the impulsivity and inattention of patients with ADHD, whereas assessments of hyperactivity currently rely on subjective reports from parents/teachers and the 
observations of clinicians. ${ }^{6}$ Diagnosing ADHD can be challenging, as the description of hyperactivity differs among informants due to its situational variability, as high activity levels are inconsistent and occur under conditions of low environmental stimulation. $^{7}$

The Quantified Behavioral Test (QbTest; Qb Tech, Stockholm, Sweden) was recently approved by the Food and Drug Administration as an effective tool to aid in the clinical assessment of ADHD. The QbTest measures the motor activity of the participant's head during a CPT test using an infrared camera. ${ }^{8}$ However, a recent study reported that the QbTest was insufficient as a diagnostic test for ADHD, as it is unable to differentiate ADHD from other neurodevelopmental disorders. ${ }^{9} \mathrm{~A}$ recent meta-analysis reported medium-to-large effect sizes using an actigraphy (standardized mean difference 0.64, 95\% confidence interval $0.43-0.85$ ) to differentiate individuals with ADHD from controls. ${ }^{4}$ However, no standardized protocol exists regarding the application of clinical actigraphy, and no normative data are available on activity according to age and gender. $^{10}$

The Robot-assisted Kinematic Measure for ADHD (RAKMA) was developed as an objective tool to assess movement in patients with ADHD. A robot assists the process to increase the engagement of the participant and detect his/her movements. The purpose of this study was to evaluate the clinical utility of the RAKMA in patients with ADHD. We hypothesized that the performance variables of the RAKMA would differentiate between individuals with ADHD and healthy controls (HCs) and that there would be significant correlations between RAKMA variables and parent-reported clinical scale scores.

\section{METHODS}

\section{Participants}

In total, 35 patients with ADHD and $50 \mathrm{HCs}$ were enrolled during December 2016 to April 2017. Patients with ADHD visited the psychiatry and pediatrics outpatient clinics of the Hanyang University Medical Center. The ADHD diagnosis was confirmed according to the criteria of the Diagnostic and Statistical Manual of Mental Disorders, Fifth Edition (DSM-5) by a thorough interview of both the child and parent administered by board-certified psychiatrists. The exclusion criteria for ADHD were: a psychiatric diagnosis other than ADHD; abnormalities in sight, hearing, or hand movement; metal allergies; and lack of cooperation. Patients with ADHD who were already taking medication were re-evaluated by a board-certified psychiatrist after a washout period of 3 days (considering that the longest half-life among ADHD medications is 6.8 hours). ${ }^{11}$ The HCs were recruited following the same exclusion criteria with the addition of a diagnosis of ADHD.

Written informed consent was obtained from all parents/ guardians, and the children provided verbal consent to participate after sufficient explanation of the study prior to enrollment. All study protocols were approved by the Institutional Review Board of the Hanyang University Medical Center (IRB Approval no. HY17-226-2). The authors assert that all procedures contributing to this study complied with the ethical standards of the relevant national and institutional committees on human experimentation and with the 2008 revision of the 1975 Declaration of Helsinki.

\section{Clinical assessments}

The CBCL is a screening tool used to measure various problem behaviors in children based on parental self-reporting; the Korean version (K-CBCL), which has well-established reliability and validity, ${ }^{12}$ consists of 120 items that are rated on a scale of 0 to $2(0=$ no behavior, $1=$ occasional behavior, and $2=$ frequent behavior). The results were transformed into T-scores with a mean of 50 and a standard deviation of 10 . Higher $\mathrm{T}$ scores indicate a greater degree of psychopathology. We used the attention problems subscale and the DSM-oriented attention problem scale scores to measure ADHD symptom severity.

ADHD symptom severity was also assessed using the Korean Attention-Deficit/Hyperactivity Disorder Diagnostic Scale (K-ADHDDS). ${ }^{13}$ The K-ADHDDS was developed based on the DSM-IV diagnostic criteria and the Gilliam Attention Deficit Hyperactivity Disorder Test. ${ }^{14}$ It consists of 36 items, with 13 items measuring hyperactivity, 10 items measuring impulsivity, and 13 items measuring inattention. Parents or teachers are instructed to rate each item on a three-point Likert scale (0-2), with higher scores indicating greater severity. The KADHDDS has an internal consistency of 0.94 and a test-retest reliability of 0.92 .

\section{The Robot-assisted Kinematic Measure for ADHD}

The robot was designed by Robocare (Seoungnam, South Korea) and was named Silbot (http://robocare.co.kr/index. $\mathrm{php} / \mathrm{silbot} /$ ) (Figure 1). The face of the robot was presented on a touch screen that showed an avatar with various facial expressions. We attached a three-dimensional (3D) camera on the chest area to detect the movements of the participants and attached LED sensors to each hand. Touch sensors and distance sensors were attached to the abdominal area to maintain distance and protect the robot from colliding with surrounding objects. We placed a 3D depth sensor around the robot to measure the participants' movement, a large-screen TV was used to present visual stimuli during the task, and a computer was used to collect movement data (Figure 2).

RAKMA is a 15-30-min test (5-10 min per trial, a total of 
3 trials) that combines a task measuring inattention and impulsivity with motor activity measurements using a robot. The robot instructs the participant to observe and then follow its

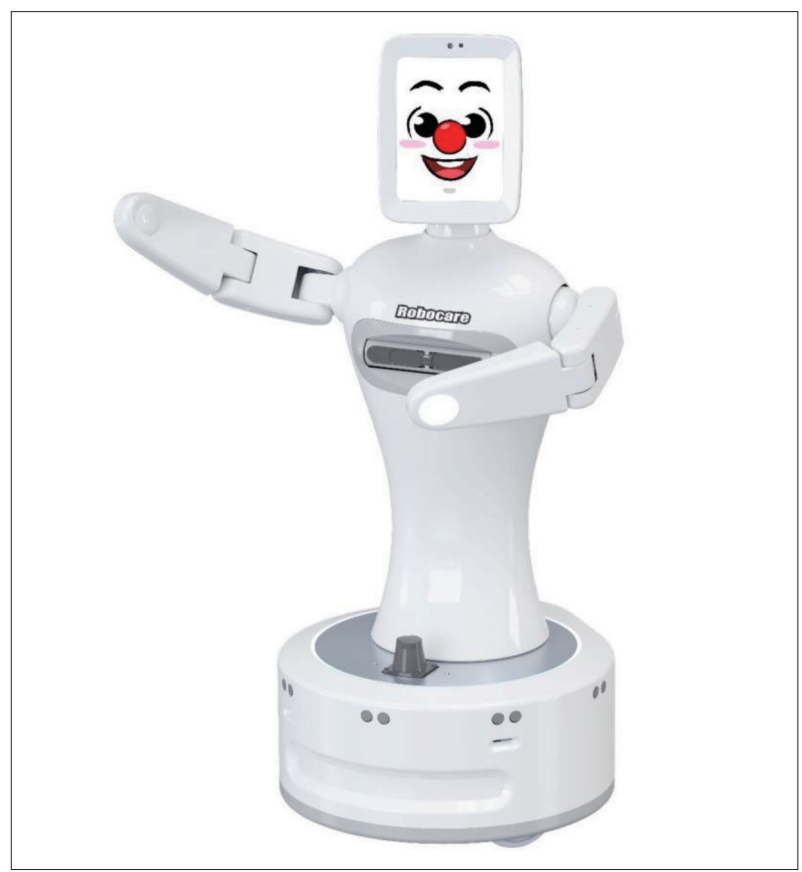

Figure 1. Silbot. movement across a $3 \times 3$ mat filled with numbers from 1 to 7 (Figure 2). As the participant moves across the mat, an auditory stimulus ("It is little red riding hood") and a visual stimulus (a person or a wolf on a TV screen) are presented simultaneously (Figure 3). When the visual stimulus is a person, the participant is instructed to wave; however, when a wolf is presented, the participant is instructed to sit down. If the participant responds correctly, a beep sound is provided, and the participant continues moving across the number mat toward the finish line. If the participant responds incorrectly, the trial ends. The participant repeats these steps three times. During trial 1 , the robot takes three steps to move from the 'start' point to the 'finish' line. During trial 2, the robot takes four steps; during trial 3, the robot takes five steps from the 'start' point to the 'finish' line. The auditory and visual stimuli were presented once in trials 1 and 2 and twice in trial 3.

We measured the stimulus-response performance based on the following four variables: 1) correct reactions (number of correct responses to simultaneous auditory and visual stimuli); 2) commission errors (number of incorrect responses to simultaneous auditory and visual stimuli, a measure of impulsivity); 3) omission errors (number of failures to respond to simultaneous auditory and visual stimuli, a measure of inattention); and 4) trial completion (number of correct responses to both

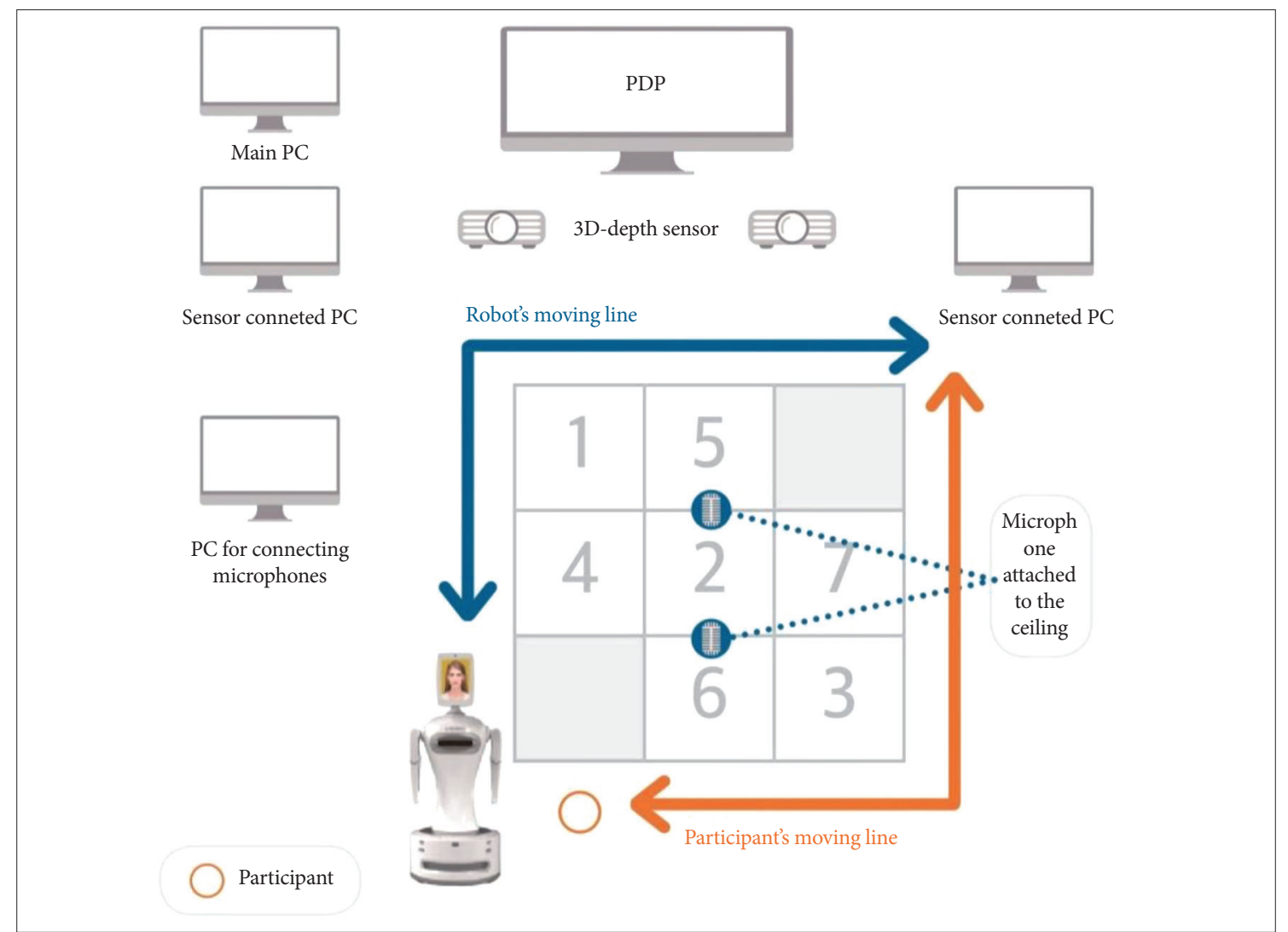

Figure 2. Diagram of the Robot-assisted Kinematic Measure for ADHD (RAKMA) system. ADHD: attention-deficit/hyperactivity disorder. 


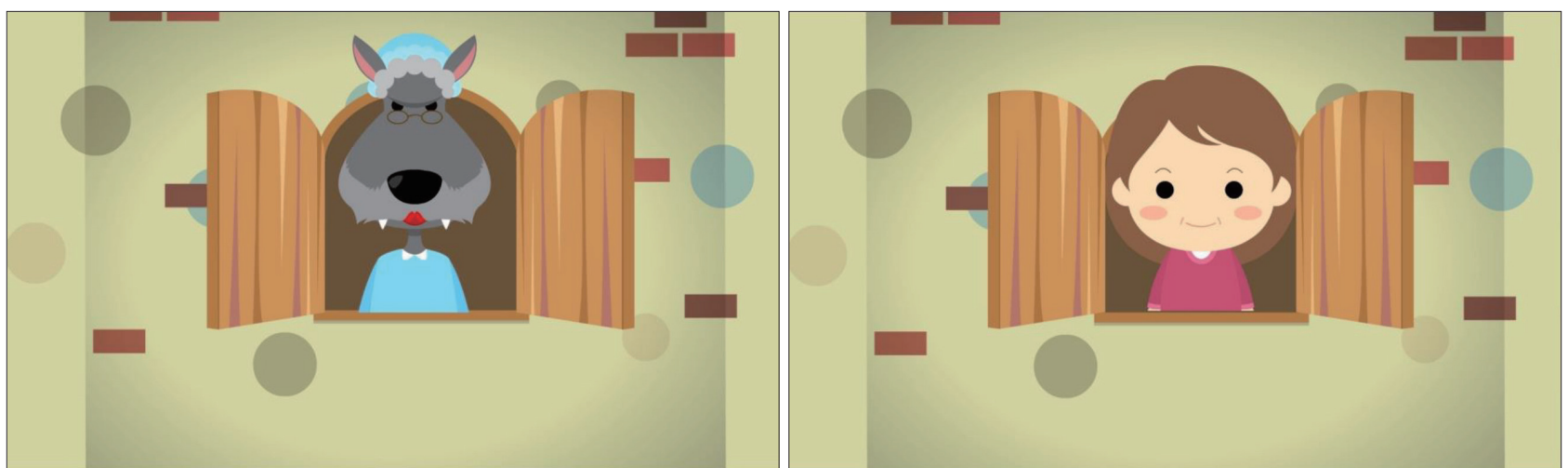

Figure 3. Pictures presented on the TV screen during the Robot-assisted Kinematic Measure for ADHD (RAKMA) task. ADHD: attentiondeficit/hyperactivity disorder.

the stimuli and the robot).

Movement was measured in terms of the following three variables: 1) reaction time (the average interval from stimulus presentation to the beep indicating a correct response during the three trials), 2) migration distance (the average difference between the participant's travelling distance and the robot's travelling distance during the three trials), and 3) migration speed (the average speed across the numbered mat during the three trials). DSM-5 defines hyperactivity as "frequent fidgeting of hands or feet, difficulties in remaining seated, inappropriate running or climbing, and/or acting as if driven by a motor." In previous studies measuring hyperactivity using actigraphy or the QbTest, hyperactivity was measured by the amount of total movement. ${ }^{4,8}$ Therefore, we defined hyperactivity as increased migration distance, which reflects to total amount of movement of the participant. Reaction time is related to processing speed and inattention, whereas migration speed would be most related to impulsivity.

\section{Statistical analysis}

The demographic and clinical characteristics of the ADHD and $\mathrm{HC}$ groups were compared using independent t-tests for continuous variables and chi-square or Fisher's exact tests for categorical variables. Due to the predominance of boys in the $\mathrm{ADHD}$ group, we compared the measures between the ADHD group and HC group in boys, separately using Mann-Whitney test. We applied Spearman's correlation analysis to investigate the correlations between ADHD clinical variables and the variables measured using the RAKMA as well as the correlation between the RAKMA stimulus-response variables and the movement variables. All statistical analyses were performed using SPSS ver. 22.0 software (IBM Corp., Armonk, NY, USA), and a p-value $<0.05$ was considered significant.

\section{RESULTS}

The demographic and clinical characteristics of the ADHD and HC groups are presented in Table 1. A higher proportion of male participants was observed in the ADHD group ( $\mathrm{p}=$ 0.014 ), and the ADHD group exhibited significantly more CBCL attention problems, DSM-oriented attention problems, and K-ADHDDS scores (all $\mathrm{p}<0.001$ ). The ADHD group had significantly fewer correct reactions, more commission errors, more omissions errors, slower reaction times, longer migration distances, and faster migration speeds.

Table 2 presents the correlations between the ADHD clinical variables and the RAKMA performance variables. Correct reactions and migration distances were significantly negatively correlated with all three clinical variables, whereas commission errors and omission errors had significant positive correlations with all three clinical variables. Migration rates were positively correlated with CBCL DSM-oriented attention problems scores as well as with K-ADHDDS scores.

Supplementary Table 1 (in the online-only Data Supplement) compares the parameters in the ADHD and $\mathrm{HC}$ groups in boys. The boys in the ADHD group showed more commission errors, longer reaction time, migration distance and also migration speed compared to the HC group. These results were similar to the main results, except that correction reactions and omission errors were no longer significant.

Table 3 shows the correlation between the RAKMA stimulus-response variables and the movement variables. Reaction times were positively correlated with trial completions. Migration distances were positively correlated with correct reactions and trial completions but negatively correlated with commission errors and omission errors. Migration speeds were negatively correlated with correct reactions but positively correlated with commission errors and omission errors.

\section{DISCUSSION}

Our results suggest that the RAKMA is clinically useful as an ADHD diagnostic aid. Patients with ADHD provided fewer correct responses and more omission errors (greater inatten- 
Table 1. Demographic and clinical characteristics of the ADHD and HC groups

\begin{tabular}{lccc}
\hline \multicolumn{1}{c}{ Characteristic } & ADHD (N=35) & HC $(\mathrm{N}=50)$ & p \\
\hline Age (years), mean (SD) & $8.8(1.5)$ & $8.7(0.9)$ & 0.934 \\
Sex (male), N (\%) & $30(85.7)$ & $23(46)$ & 0.014 \\
Grade, N (\%) & $2.3(1.4)$ & $2.9(0.9)$ & 0.776 \\
CBCL, attention problems score, mean (SD) & $63.4(7.6)$ & $2.8(3.9)$ & $<0.001$ \\
CBCL, DSM-oriented attention problems score, mean (SD) & $67.8(11.1)$ & $53.6(5.3)$ & $<0.001$ \\
K-ADHDDS score, mean (SD) & $25.4(6.3)$ & $12.4(3.4)$ & $<0.001$ \\
RAKMA variables, mean (SD) & & & \\
Correct reactions (N) & $2.0(1.5)$ & $2.7(1.2)$ & 0.015 \\
Commission errors (N) & $0.7(0.8)$ & $0.2(0.5)$ & $<0.001$ \\
Omission errors (N) & $0.9(1.2)$ & $0.4(0.8)$ & 0.028 \\
Trial completion (N) & $1.6(1.1)$ & $1.4(1.1)$ & 0.343 \\
Reaction time (s) & $2.7(2.5)$ & $1.7(1.1)$ & 0.010 \\
Migration distance (m) & $0.8(6.3)$ & $5.0(4.2)$ & $<0.001$ \\
Migration speed (m/s) & $0.7(1.2)$ & $0.4(0.1)$ & 0.035 \\
\hline
\end{tabular}

ADHD: attention-deficit/hyperactivity disorder, HC: healthy control, SD: standard deviation, CBCL: Child Behavior Checklist, DSM: Diagnostic and Statistical Manual of Mental Disorders, K-ADHDDS: Korean Attention-Deficit/Hyperactivity Disorder Diagnostic Scale, RAKMA: Robot-assisted Kinematic Measure for ADHD

Table 2. Correlation between ADHD clinical scale scores and RAKMA task performance variables

\begin{tabular}{lccc}
\hline & CBCL attention problems score & CBCL DSM-oriented attention problems score & K-ADHDDS score \\
\hline Correct reactions (N) & $-0.335^{\dagger}$ & $-0.347^{\dagger}$ & $-0.327^{\dagger}$ \\
Commission errors (N) & $0.327^{\dagger}$ & $0.345^{\dagger}$ & $0.334^{\dagger}$ \\
Omission errors (N) & $0.310^{\dagger}$ & $0.311^{\dagger}$ & $0.349^{\dagger}$ \\
Trial completion (N) & -0.090 & -0.084 & -0.004 \\
Reaction time (s) & 0.051 & 0.086 & 0.036 \\
Migration distance (m) & $-0.306^{\dagger}$ & $-0.356^{\dagger}$ & $-0.426^{\dagger}$ \\
Migration rate (m/s) & 0.158 & $0.335^{\dagger}$ & $0.240^{*}$ \\
\hline
\end{tabular}

${ }^{*} \mathrm{p}<0.05,{ }^{\dagger} \mathrm{p}<0.01$. ADHD: attention-deficit/hyperactivity disorder, RAKMA: Robot-assisted Kinematic Measure for ADHD, CBCL: Child Behavior Checklist, DSM: Diagnostic and Statistical Manual of Mental Disorders, K-ADHDDS: Korean Attention-Deficit/Hyperactivity Disorder Diagnostic Scale, n: numbers, s: seconds, m: meters, m/s: meters per second

Table 3. Correlation between RAKMA stimuli response variables and movement variables

\begin{tabular}{lcccc}
\hline & Correct reaction $(\mathrm{N})$ & Commission errors (N) & Omission errors (N) & Trial completion $(\mathrm{N})$ \\
\hline Reaction time $(\mathrm{s})$ & 0.169 & -0.004 & -0.196 & $0.610^{\dagger}$ \\
Migration distance $(\mathrm{m})$ & $0.468^{\dagger}$ & $-0.531^{\dagger}$ & $-0.299^{\dagger}$ & $0.417^{\dagger}$ \\
Migration speed (m/s) & $-0.280^{\dagger}$ & $0.372^{\dagger}$ & $0.361^{*}$ & 0.007 \\
\hline
\end{tabular}

${ }^{*} \mathrm{p}<0.05,{ }^{\dagger} \mathrm{p}<0.01$. RAKMA: Robot-assisted Kinematic Measure for attention-deficit/hyperactivity disorder, n: numbers, s: seconds, m: meters, $\mathrm{m} / \mathrm{s}$ : meters per second

tion) and commission errors (greater impulsivity) than the HCs, but there were no differences in trial completions. Patients with $\mathrm{ADHD}$ also had slower reaction times, greater migration distances, and faster migration speeds, which indicates higher levels of inattention, hyperactivity, and impulsivity, respectively. Trial completions and reaction times were not correlated with the clinical scale scores, suggesting that these two variables are less useful than the other RAKMA variables for diagnosing
ADHD. Migration distance, a measure of hyperactivity, was significantly correlated with clinical scores rated by the parents, suggesting high criterion validity. The neuropsychological performance of patients with ADHD can be confounded by their diagnosis. ${ }^{15}$ Barkley ${ }^{16}$ hypothesized that hyperactive patients would perform worse on executive tasks, such as those involving delayed reactions, problem solving, flexibility, and sustained attention, whereas inattentive patients would be more 
impaired on tasks involving memory, focused attention, and timed information processing. Another study reported that inattentive children have more problems with selective attention and slow processing, whereas hyperactive children have more difficulties with executive and inhibitory functions. ${ }^{17}$ Barkley ${ }^{18}$ postulated that hyperactivity is connected to dopaminergic dysfunction whereas hypoactivity is a norepinephrine phenomenon. These changes are accompanied by different neuropsychological profiles. Therefore, objective measures of both hyperactivity and neuropsychological functioning (e.g., the RAKMA) may be more useful than simple neuropsychological function tests for differentiating among ADHD subtypes. Future studies with sample sizes large enough to conduct subanalyses according to ADHD subtype are warranted.

Previous infrared-based motion-tracking tools were limited by the fact that they measured only head movement. The McLean Motion and Attention Test System, a precursor to the Qb test, measures head immobility duration, head movement, head displacement, head area, head spatial complexity, and head temporal scaling. ${ }^{19}$ The $\mathrm{Qb}$ test presents data on head movement using five raw parameters: time active, distance, area, microevents, and motion simplicity. ${ }^{8}$ An actigraph measures movement on only the part of the body on which the actigraph is applied. The RAKMA is unique, as it tracks the movements of the whole body during the 10-min task. However, there is no gold standard regarding the area of body measurement. Further studies should be conducted to examine which regions of the body are best to measure when determining hyperactivity in patients with ADHD.

Another strength of the RAKMA is its use of a robot to assist the patient with the task. Research on the use of socially assistive robotics (SAR) in child psychiatry has been mainly focused on autism spectrum disorder (ASD), and the application of SAR to ADHD is novel. ${ }^{20}$ SAR refers to a robot that provides assistance to the user in social interactions. ${ }^{21}$ SAR is useful for eliciting target behavior for purposes of diagnosis and treatment, modeling and teaching skills, and quantifying observed behavior in children with ASD. SAR has also been demonstrated to improve cognitive attention and cortical neuron activity and has the ability to alleviate stress in patients with dementia. ${ }^{22}$ Moreover, as patients needed less supervision while interacting with the robot, their caregivers experienced less stress. This is the first study to apply robotic techniques to diagnose ADHD.

Our study had several limitations. First, the sample size was small, limiting statistical power. A significant difference in gender distribution was observed between the ADHD and HC groups, and there were only five female participants in the ADHD group, which could have confounded the results. However, a subgroup analysis of boys rendered similar results to that of both genders combined. We included all subtypes of ADHD due to the sam- ple size limitation, making it a heterogeneous group with variable behavioral characteristics. We did not perform an IQ test and therefore could not exclude children with intellectual disabilities. Further studies should examine RAKMA performance according to subtype to reduce the confounding effect of subtype.

\section{Supplementary Materials}

The online-only Data Supplement is available with this article at https://doi.org/10.30773/pi.2021.0036.

\section{Acknowledgments}

This study was supported by a fund from the Ministry of Trade, Industry, and Energy of South Korea (No. 20003762 to D.-H.A.) and by the Bio \& Medical Technology Development Program of the National Research Foundation (NRF) \& funded by the Korean Government (MSIT) (2019M3E5D1A01069345 to J.-I.K.).

\section{Conflicts of Interest}

The authors have no potential conflicts of interest to disclose.

\section{Author Contributions}

Conceptualization: Hak Jong Noh, Dong Hyun Ahn. Data curation: Ga Hyun Lee, Seong-kyu Kang. Funding acquisition: Hak Jong Noh, Moon Sang Kim, Hyo-Shin Lee, Mun-Taek Choi, Dong Hyun Ahn. Investigation: Ga Hyun Lee, Aran Min. Methodology: Aran Min, Johanna Inhyang Kim. Resources: Kyuha Lee, Jun-Ho Seo. Software: Moon Sang Kim. Supervision: Dong Hyun Ahn. Writing_-original draft: Aran Min, Johanna Inhyang Kim. Writing—review \& editing: Johanna Inhyang Kim.

\section{ORCID iDs}

Aran Min

Johanna Inhyang Kim

Hak Jong Noh

Moon Sang Kim

Hyo-Shin Lee

Mun-Taek Choi

Kyuha Lee

Jun-Ho Seo

Ga Hyun Lee

Seong-kyu Kang

Dong Hyun Ahn
https://orcid.org/0000-0002-0450-739X https://orcid.org/0000-0002-2367-0934 https://orcid.org/0000-0002-3386-6252 https://orcid.org/0000-0002-6050-6594 https://orcid.org/0000-0003-3298-5548 https://orcid.org/0000-0002-5025-8785 https://orcid.org/0000-0002-9634-2640 https://orcid.org/0000-0003-0330-1805 https://orcid.org/0000-0002-3736-9616 https://orcid.org/0000-0003-3735-7102 https://orcid.org/0000-0002-9114-7790

\section{REFERENCES}

1. American Psychiatric Association. Diagnostic Statistical Manual of Mental Disorders: DSM-5. 5th Ed. Washington, DC: American Psychiatric Association; 2013.

2. Lange KW, Reichl S, Lange KM, Tucha L, Tucha O. The history of attention deficit hyperactivity disorder. Atten Defic Hyperact Disord 2010;2:241-255.

3. World Health Organization. ICD-10 Classifications of Mental and Behavioral Disorder: Clinical Descriptions and Diagnostic Guidelines. Geneva: World Health Organization; 1992.

4. De Crescenzo F, Licchelli S, Ciabattini M, Menghini D, Armando M, Alfieri P, et al. The use of actigraphy in the monitoring of sleep and activity in ADHD: a meta-analysis. Sleep Med Rev 2016;26:9-20.

5. Wood AC, Asherson P, Risjsdijk F, Kuntsi J. Is overactivity a core feature in $\mathrm{ADHD}$ ? Familial and receiver operating characteristic curve analysis of mechanically assessed activity level. J Am Acad Child Adolesc Psychiatry 2009;48:1023-1030.

6. Valo S, Tannock R. Diagnostic instability of DSM-IV ADHD subtypes: effects of informant source, instrumentation, and methods for combin- 
ing symptom reports. J Clin Child Adolesc Psychol 2010;39:749-760.

7. Antrop I, Roeyers H, Van Oost P, Buysse A. Stimulation seeking and hyperactivity in children with ADHD. Attention deficit hyperactivity disorder. J Child Psychol Psychiatry 2000;41:225-231.

8. Hult N, Kadesjo J, Kadesjo B, Gillberg C, Billstedt E. ADHD and the QbTest: diagnostic validity of QbTest. J Atten Disord 2018;22:10741080.

9. Johansson V, Noren Selinus E, Kuja-Halkola R, Lundstrom S, Durbeej $\mathrm{N}$, Anckarsater $\mathrm{H}$, et al. The quantified behavioral test failed to differentiate ADHD in adolescents with neurodevelopmental problems. J Atten Disord 2021;25:312-321.

10. Miyahara M, Healey DM, Halperin JM. One-week temporal stability of hyperactivity in preschoolers with ADHD during psychometric assessment. Psychiatry Clin Neurosci 2014;68:120-126.

11. Gibaldi M. Biopharmaceutics and Clinical Pharmacokinetics. 3rd Ed. Philadelphia, USA: Lea and Febiger; 1984.

12. Oh K, Lee H, Hong K, Ha E. Korean Version of Child Behavior Checklist (K-CBCL). Seoul, Korea: Chungang Aptitude Publishing Co.; 1997.

13. Yoon CY, Lee YS. A normative study of Korean-Attention Deficit Hyperactivity Disorder Diagnostic Scale and developing Web-based computer assessment system. Kor J Counsel 2004;6:59-73.

14. Gilliam JE. Attention-Deficit/Hyperactivity Disorder Test: A Method for Identifying Individuals with ADHD. Austin, Texas: Pro-ed; 1995.

15. Schoechlin C, Engel RR. Neuropsychological performance in adult at- tention-deficit hyperactivity disorder: meta-analysis of empirical data. Arch Clin Neuropsychol 2005;20:727-744.

16. Barkley RA. Behavioral inhibition, sustained attention, and executive functions: constructing a unifying theory of ADHD. Psychol Bull 1997; 121:65-94.

17. Lockwood KA, Marcotte AC, Stern C. Differentiation of attention-deficit/hyperactivity disorder subtypes: application of a neuropsychological model of attention. J Clin Exp Neuropsychol 2001;23:317-330.

18. Barkley RA. Attention-Deficit Hyperactivity Disorder: A Handbook for Diagnosis and Treatment. 2nd Ed. New York: The Guildford Press; 1990.

19. Teicher MH, Ito Y, Glod CA, Barber NI. Objective measurement of hyperactivity and attentional problems in ADHD. J Am Acad Child Adolesc Psychiatry 1996;35:334-342.

20. Pennisi P, Tonacci A, Tartarisco G, Billeci L, Ruta L, Gangemi S, et al. Autism and social robotics: a systematic review. Autism Res 2016;9: 165-183.

21. Cho SJ, Ahn DH. Socially assistive robotics in autism spectrum disorder. Hanyang Med Rev 2016;36:17-26.

22. Góngora Alonso S, Hamrioui S, de la Torre Díez I, Motta Cruz E, López-Coronado M, Franco M. Social robots for people with aging and dementia: a systematic review of literature. Telemed J E Health 2019; 25:533-540. 
Supplementary Table 1. Comparison of RAKMA parameters between ADHD and $\mathrm{HC}$ groups in boys only

\begin{tabular}{lccc}
\hline \multicolumn{1}{c}{ RAKMA variables } & ADHD $(\mathrm{N}=30)$ & $\mathrm{HC}(\mathrm{N}=23)$ & $\mathrm{p}$ \\
\hline Correct reactions $(\mathrm{N})$ & 24.5 & 30.2 & 0.170 \\
Commission errors $(\mathrm{N})$ & 31.3 & 21.4 & 0.007 \\
Omission errors $(\mathrm{N})$ & 28.3 & 25.3 & 0.419 \\
Reaction time $(\mathrm{s})$ & 30.3 & 21.7 & 0.04 \\
Migration distance $(\mathrm{m})$ & 42.2 & 35.9 & $<0.001$ \\
Migration speed $(\mathrm{m} / \mathrm{s})$ & 31.7 & 20.9 & 0.012 \\
\hline
\end{tabular}

ADHD: attention-deficit/hyperactivity disorder, HC: healthy control, RAKMA: Robot-assisted Kinematic Measure for ADHD 